\title{
Segond fracture with anterior cruciate ligament tear in an adolescent
}

\author{
F. Falciglia $\cdot$ G. Mastantuoni $\cdot$ V. Guzzanti
}

Received: 1 July 2008/ Accepted: 23 July 2008/Published online: 16 August 2008

(C) Springer-Verlag 2008

\begin{abstract}
The authors report a case of acute knee injury in a 14-year-old teenager. The X-ray showed a so-called Segond's fracture: a small avulsed bone fragment, elliptical in shape, lying immediately below the external tibial plateau, a few millimeters from the lateral tibial cortex. The fracture site was in the portion of the tibial condyle which is linked to the middle third of the lateral capsule by meniscal tibial fibers. Clinical examination under anesthesia and subsequent arthroscopy revealed a total intrasubstance ACL (anterior cruciate ligament) tear close to the proximal insertion. The authors confirm Segond's report of a possible association of this avulsion fracture with ACL injuries, even in adolescence.
\end{abstract}

Keywords Segond fracture ACL tear - Knee . Avulsion fracture

\section{Introduction}

Over a century ago, in 1879, Segond [1] described a small fracture located on the tibial lateral capsular ligament insertion and posterior to Gerdy's tubercle. Segond produced this avulsion fracture fragment experimentally by applying traction forces to the anterolateral ligamentous compartment (by internal rotation of the tibia with the knee flexed). From a clinical point of view, this fracture is

F. Falciglia $(\bowtie) \cdot$ G. Mastantuoni · V. Guzzanti Orthopaedics and Traumatology Division, Bambino Gesù

Children's Hospital, Institute of Scientific Research,

Piazza San Onofrio 4, 00165 Rome, Italy

e-mail: falciglia@opbg.net

V. Guzzanti

University of Cassino, Cassino (FR), Italy important, as it is often combined with other injuries: tear of the anterior cruciate ligament (ACL) at the site of femoral attachment, avulsion fragmentation of the fibular collateral ligament at its distal attachment, and, more rarely, sprain of the tibiofibular joint. Although the first radiographic observation of the so-called Segond's fracture dates back to 1936 [2], it is only recently [3-7] that the importance of identifying this fracture with an X-ray has been emphasized, in order to ensure a more accurate assessment of the external instability of the knee. There is only one report of such a case observed in children and adolescents [8]. Some authors [3, 7] have underlined the need for a routine X-ray examination, as well as the accurate clinical examination with the usual static and dynamic tests that is also obviously required, whenever an injured knee is under observation. In this way, a clearer and more immediate evaluation of the injury can be obtained. In particular, many avulsed bone fragments can be observed either singularly or in combination with others in the lateral compartment of the knee (LCL avulsion fracture, avulsion fracture of the fibular head, detachment of the fibular collateral ligament from its femoral or fibular attachment, avulsion fracture of the popliteus tendon at its femoral attachment site, Gerdy's tubercle avulsion fracture at the insertion of the iliotibial tract). These findings must be researched and assessed differently, according to what they suggest, each time a patient with clinical signs of a lateral capsular ligament injury is examined-even in children and adolescents.

\section{Case report}

The patient was a 14-year-old girl under observation for an acute knee injury. The history of the accident (it occurred 
during a volleyball match at school) explained the mechanism of injury: an axial load on a semi-flexed knee with a rotational component [9]. Clinical examination revealed a marked swelling of the knee. On physical examination, the patient referred to acute pain in the external compartment just below the lateral tibial joint surface immediately before the fibular head. The hemarthrosis $(50 \mathrm{cc})$ was aspirated. On admission, the Lachman test and the passive anterior drawer test were slightly positive, even if they were difficult to perform because of pain. X-ray of the right knee showed a small bone fragment on the anterior-posterior and oblique views, elliptical in outline, near the lateral tibial condylar cortex, just below the articular surface (Fig. 1a). The avulsion fracture had occurred at the site of the tibial condyle that is linked to the middle third of the lateral joint capsule by the meniscal tibial fibers.

Dynamic X-ray under anesthesia did not show a marked passive anterior drawer of the right tibia compared to the controlateral side, with Lachman and pivot shift tests slightly positive. During arthroscopy, other intra-articular injuries were excluded and a total intrasubstance ACL tear at its proximal insertion was observed (Fig. 1b). Immobilization followed with a functional knee brace for 3 weeks and partial weight-bearing for another three weeks. The patient was available for review at 17 years after injury. The assessment included a questionnaire on symptoms and function, a clinical examination, and a measurement of the laxity in the sagittal plane. At follow-up, the passive anterior drawer sign, the Lachman and the pivot shift tests were slightly positive. The patient was free of the symptoms 17 years after the initial injury, but she avoided strenuous sports activities.
The patient provided her consent for the publication of this case report.

\section{Discussion}

Present knowledge of the pathogenesis of capsular ligamentous knee injuries requires an assessment that is as complete as possible and is made as early as possible of any tears, in order to avoid the possibility that a late or insufficient diagnosis might lead to functional instability and progressive joint damage. This may result in poor function and degenerative changes, especially in patients engaged in strenuous activities.

The above is even important in children and adolescents, where, due to intense sport activities, observations of cases of ACL tear are becoming more and more common. The LCL avulsion fracture was first described by Segond before the discovery of $\mathrm{X}$-rays by Roentgen, stressing the importance of its combination with other injuries (especially ACL tears). According to some authors [3], this fracture is a sign of a lateral damage and can be associated with a total ACL disruption in a high percentage of cases (75-100\%).

In adolescents, the appearance of Segond fracture has not been previously reported in association with ACL intrasubstance tear. Only Sferopoulos [8] has reported cases of Segond fracture in children and adolescents previously, but these were associated with avulsed intercondylar eminence. Anatomical and biomechanical reasons explain why a bone avulsion at the tibial insertion of LCL occurs instead of a tear of the ligament itself. In fact, at the middle third of
Fig. 1 a M.C., 14 years old. "Segond fracture:" an avulsed bone fragment of the proximal tibial condyle, below the articular surface at the insertion of LCL. b Arthroscopic view of the ACL tear

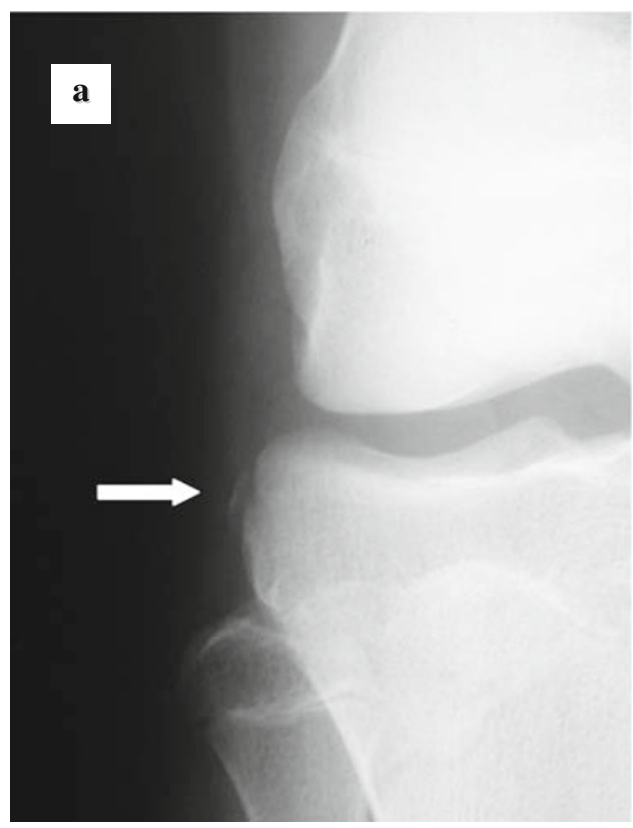


Fig. 2 Drawings of Segond's fracture and associated types of ACL injuries

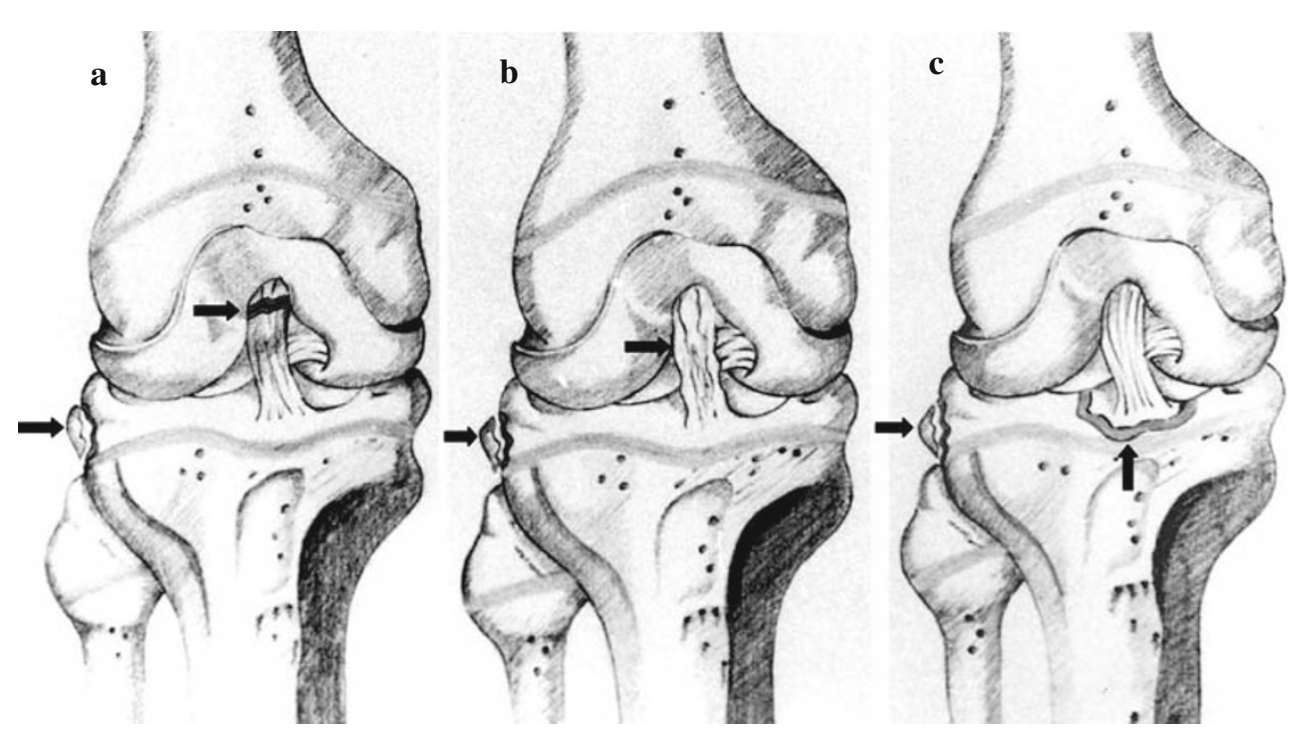

the lateral knee structure, the deep, the intermediate and the superficial layers consist respectively of two, strongly fused, capsular laminae and the iliotibial tract. This reinforced anatomical structure of the LCL makes avulsion more likely than ligament tear. Upon examining the Xrays, we must consider that there may be other findings of lateral ligamentous damage to the knee that may be confused for Segond fracture and that may be present alone or associated with it (Gerdy's tubercle avulsion fracture from the iliotibial tract, the avulsed fragment of the fibular head at the site of insertion of the biceps femoris tendon or of the fibular collateral ligament, and osteochondral fragment avulsion from the popliteus tendinous femoral insertion).

In conclusion, although an accurate patient hystory and a careful clinical examination can lead us to a correct diagnosis of capsular ligamentous injuries of the knee (which is difficult, even in the acute phase), X-rays should always be taken of patients with knee injuries, even children and adolescents. Segond fracture must always be looked for, because it often indicates peripheral damage and possible ACL involvement-total or partial rupture or avulsion (Fig. 2a-c). In the above reported case of a 14-year-old girl, this association was confirmed by both clinical and arthroscopic exams. The choice of following conservative or surgical treatment depends on the laxity, the functional instability, and on the demands of the sporting and vigorous activities that will be engaged in. In our case, conservative treatment resulted in a subjective satisfactory result at
17 years of follow-up, probably because the patient avoided strenuous activities.

Conflict of interest statement The authors declare that they have no conflict of interest related to the publication of this manuscript.

\section{References}

1. Segond $P$ (1979) Recherches cliniques et expèrimentales sur les èpanchements sanguins du genou par entorse. Progrès Méd 7:297299, 319-321, 340-341,379-381, 400-401, 419-421

2. Milch $H$ (1936) Cortical avulsion fracture of the lateral tibial condyle. J Bone Joint Surg 18:159-164

3. Dietz GW, Wilcox DM, Montgomery JB (1986) Segond tibial condyle fracture: lateral capsular ligament avulsion. Radiology 159:467-469

4. Johnson LL (1979) Lateral capsular ligament complex: anatomical and surgical considerations. Am J Sport Med 7:156-160

5. Li D, Flak B (1991) Segond knee injury in a skier. Clin J Sport Med 1:41-42

6. Norwood LA, Andrewss JR, Meisterling RC, Glancy GL (1979) Acute anterolateral rotatory instability of the knee. J Bone Joint Surg Am 61:704-709

7. Woods GW, Stanley RF, Tullos HS (1979) Lateral capsular sign: X-ray clue to a significant knee instability. Am J Sport Med 1:2733

8. Sferopoulos NK, Rafailidis D, Traios S, Christoforides J (2006) Avulsion fractures of the lateral tibial condyle in children. Injury 37(1):57-60

9. Irvine GB, Dias JJ, Finlay DB (1987) Segond fracture of the lateral tibial condyle: brief report. J Bone Joint Surg Br 69:613-614 